\title{
Quantitative and Qualitative Studies of Science and Technology in Latin America
}

\author{
Guest Editor:
}

Claudia N. González Brambila 